\title{
Molecular Imaging of PD-L1 Expression and Dynamics with the Adnectin-Based PET Tracer ${ }^{18}$ F-BMS-986192
}

\author{
Thijs S. Stutvoet*1, Elly L. van der Veen*1, Arjan $\mathrm{Kol}^{1}$, Inês F. Antunes ${ }^{2}$, Erik F.J. de Vries ${ }^{2}$, Geke A.P. Hospers ${ }^{1}$, \\ Elisabeth G.E. de Vries ${ }^{1}$, Steven de Jong ${ }^{1}$, and Marjolijn N. Lub-de Hooge ${ }^{2,3}$ \\ ${ }^{1}$ Departments of Medical Oncology, University Medical Center Groningen, University of Groningen, Groningen, The Netherlands; \\ ${ }^{2}$ Nuclear Medicine and Molecular Imaging, University Medical Center Groningen, University of Groningen, Groningen, The \\ Netherlands; and ${ }^{3}$ Clinical Pharmacy and Pharmacology, University Medical Center Groningen, University of Groningen, \\ Groningen, The Netherlands
}

\begin{abstract}
${ }^{18} \mathrm{~F}-\mathrm{BMS}-986192$, an adnectin-based human programmed cell death ligand 1 (PD-L1) tracer, was developed to noninvasively determine whole-body PD-L1 expression by PET. We evaluated the usability of ${ }^{18} \mathrm{~F}$-BMS-986192 PET to detect different PD-L1 expression levels and therapy-induced changes in PD-L1 expression in tumors. Methods: In vitro binding assays with ${ }^{18} \mathrm{~F}-\mathrm{BMS}-986192$ were performed on human tumor cell lines with different total cellular and membrane PD-L1 protein expression levels. Subsequently, PET imaging was performed on immunodeficient mice xenografted with these cell lines. The mice were treated with interferon y (IFNy) intraperitoneally for $3 \mathrm{~d}$ or with the mitogen-activated protein kinase kinase inhibitor selumetinib by oral gavage for $24 \mathrm{~h}$. Afterward, ${ }^{18} \mathrm{~F}-$ BMS-986192 was administered intravenously, followed by a 60 -min dynamic PET scan. Tracer uptake was expressed as percentage injected dose per gram of tissue. Tissues were collected to evaluate ex vivo tracer biodistribution and to perform flow cytometric, Western blot, and immunohistochemical tumor analyses. Results: ${ }^{18} \mathrm{~F}-$ BMS-986192 uptake reflected PD-L1 membrane levels in tumor cell lines, and tumor tracer uptake in mice was associated with PD-L1 expression measured immunohistochemically. In vitro IFNy treatment increased PD-L1 expression in the tumor cell lines and caused up to a 12-fold increase in tracer binding. In vivo, IFNy affected neither PD-L1 tumor expression measured immunohistochemically nor ${ }^{18}$ F-BMS-986192 tumor uptake. In vitro, selumetinib downregulated cellular and membrane levels of PD-L1 in tumor cells by $50 \%$ as measured by Western blotting and flow cytometry. In mice, selumetinib lowered cellular, but not membrane, PD-L1 levels of tumors, and consequently, no treatment-induced change in ${ }^{18} \mathrm{~F}-\mathrm{BMS}-$ 986192 tumor uptake was observed. Conclusion: ${ }^{18} \mathrm{~F}-\mathrm{BMS}-$ 986192 PET imaging allows detection of membrane-expressed PD-L1 as soon as 60 min after tracer injection. The tracer can discriminate a range of tumor cell PD-L1 membrane expression levels.
\end{abstract}

Key Words: molecular imaging; PET; programmed cell death ligand 1; ${ }^{18}$ F-labeled adnectin; ${ }^{18}$ F-BMS-986192

J Nucl Med 2020; 61:1839-1844

DOI: 10.2967/jnumed.119.241364

Received Jan. 9, 2020; revision accepted Mar. 31, 2020.

For correspondence or reprints contact: Marjolijn N. Lub-de Hooge, Department of Clinical Pharmacy and Pharmacology, University Medical Center Groningen, P.O. Box 30.001, 9700 RB Groningen, The Netherlands. E-mail: m.n.de.hooge@umcg.nl

*Contributed equally to this work.

Published online May 1, 2020.

COPYRIGHT (C 2020 by the Society of Nuclear Medicine and Molecular Imaging.
$\mathbf{P}$ death ligand 1 (PD-L1) inhibitors have radically improved treatment of patients with cancer. These drugs have been approved for treatment of many tumor types and for unresectable and microsatellite instable and mismatch repair deficient metastatic solid tumors (1). Combining a PD-1 antibody with the cytotoxic T-lymphocyte-associated protein 4 antibody ipilimumab increases efficacy in melanoma (2). In addition, numerous studies aim to improve efficacy by combining PD-1/PD-L1 antibodies with novel immune checkpoint inhibitors, targeted agents, chemotherapies, and radiotherapy (3).

Despite impressive antitumor effects, many patients do not respond to PD-1/PD-L1-targeted treatment. Still, these patients are at risk for side effects $(4,5)$. To select patients who are most likely to respond to immune checkpoint inhibitors, immunohistochemical quantification of tumor cell PD-L1 expression can be performed (6). However, discrepancy between PD-L1 status and tumor response occurs $(7,8)$. Immunohistochemical analysis of a single tumor-biopsy sample may fail to capture the heterogeneity of PD-L1 expression within and between lesions or the change in expression over time (9-12).

PET is a potential method to noninvasively evaluate whole-body PD-L1 expression levels. Radiolabeled antibodies targeting PD-1 or PD-L1 have been used in this context $(7,13,14)$. Imaging with radiolabeled antibodies requires several days of tracer clearance from circulation to obtain PET images with adequate contrast (13). Given their fast tumor penetration and short serum halflives, radiolabeled small molecules targeting PD-L1 could yield adequate contrast for imaging within $1 \mathrm{~h}$, allowing imaging on the day of tracer injection (7). The approximately $12-\mathrm{kDa}$ adnectin-based human PD-L1-targeting PET tracer ${ }^{18} \mathrm{~F}-\mathrm{BMS}$ 986192 has been developed for this purpose (15). In preclinical studies, ${ }^{18}$ F-BMS-986192 imaging allowed distinction of a PDL1-positive from a PD-L1-negative tumor within $2 \mathrm{~h}$ after tracer injection (16).

Here, we aimed to evaluate whether ${ }^{18} \mathrm{~F}-\mathrm{BMS}-986192$ PET can be used to distinguish different intrinsic PD-L1 expression levels in tumor cell lines and tumor xenografts in mice. Moreover, we investigated whether ${ }^{18} \mathrm{~F}-\mathrm{BMS}-986192$ PET can be used to detect therapy-induced modulation of PD-L1 expression levels. Ex vivo analysis of tumor tissue using flow cytometry, Western blotting, and immunohistochemistry was performed to evaluate PD-L1 expression levels. 


\section{MATERIALS AND METHODS}

\section{Cell Lines and Reagents}

The human tumor cell lines H292 (lung mucoepidermoid carcinoma) and H358 (lung adenocarcinoma) were obtained from the American Type Culture Collection, H322 (human lung adenocarcinoma) was obtained from Sigma-Aldrich, and ES2 (human ovarian clear cell carcinoma) was a kind gift from Dr. Els Berns (Erasmus University Medical Center). All cells were cultured in RPMI 1640 (Invitrogen) medium with $10 \%$ fetal calf serum (Bodinco BV) (supplemented with $2 \mathrm{mM} \mathrm{L}$-glutamine for $\mathrm{H} 322$ cells) and maintained in a humidified atmosphere with $5 \% \mathrm{CO}_{2}$ at $37^{\circ} \mathrm{C}$. Cells were regularly tested for Mycoplasma contamination and were proven to be Mycoplasma-negative. Cell line authentication was regularly performed using short tandem repeat profiling.

\section{Tracer Production}

The human PD-L1-specific PET tracer ${ }^{18}$ F-BMS-986192 was produced with a radiochemical purity of more than $90 \%$ and molar activity of more than $6,100 \mathrm{GBq} / \mathrm{mmol}$ according to a slightly modified version of a previously published protocol (supplemental methods (16); supplemental materials are available at http://jnm.snmjournals. org). In short, ${ }^{18} \mathrm{~F}-\mathrm{BMT}-187144$ is formed by fluorination of the precursor BMT-180478-01 (Bristol-Myers Squibb) with ${ }^{18} \mathrm{~F}$-fluoride. Next, ${ }^{18} \mathrm{~F}-\mathrm{BMS}-986192$ is generated by a [2,3]-cycloaddition reaction of the cyclooctyne moiety in the anti-PD-L1 adnectin precursor BMT-192920 (Bristol-Myers Squibb) with the azide group in ${ }^{18}$ F-BMT-187144. Ultrahigh-performance liquid chromatography was used to determine chemical and radiochemical purity, radiochemical identity, and molar activity (supplemental methods). In vitro and in vivo tracer stability were demonstrated previously (16).

\section{Tracer-Binding Studies}

For binding assays, $0.1 \times 10^{6}$ cells were grown for $24 \mathrm{~h}$ in RPMI medium with $10 \%$ fetal calf serum in 24-well plates and treated with the inducer of PD-L1, interferon- $\gamma$ (IFN $\gamma$; R\&D systems), with a final concentration up to $6 \times 10^{5} \mathrm{IU} / \mathrm{mL}(30 \mathrm{ng} / \mathrm{mL}$, diluted in sterile water) (17). Tracer (1 MBq, $50 \mu \mathrm{L}, 3,330 \mathrm{ng} / \mathrm{mL}, 167 \mathrm{ng}$ ) was added to each well, and cells were incubated for $60 \mathrm{~min}$ at $37^{\circ} \mathrm{C}$. Competition assays were performed by adding $50 \mu \mathrm{L}$ of a mixture of $1,400 \mathrm{ng} / \mathrm{mL}$ tracer solution with increasing amounts of nonradioactive ${ }^{19} \mathrm{~F}-\mathrm{BMS}-986192$ (from $5 \mathrm{ng} / \mathrm{mL}$ to $4 \times 10^{5} \mathrm{ng} / \mathrm{mL}$ ) to each well. After incubation, the cells were washed twice with $1 \mathrm{~mL}$ of ice-cold phosphate-buffered saline ([PBS] $9.7 \mathrm{mM} \mathrm{Na}_{2} \mathrm{HPO}_{4}, 1.6 \mathrm{mM} \mathrm{KH}_{2} \mathrm{PO} 4,150 \mathrm{mM} \mathrm{NaCl}, \mathrm{pH}$ 7.2) containing $1 \%$ human serum albumin. The cells were trypsinized, and medium was added. Cell suspensions were transferred to plastic tubes. Radioactivity in the cell fraction was measured in a $\gamma$-counter (Wizard ${ }^{2}$ 2480-0019, SW 2.1; PerkinElmer). To correct for IFN $\gamma$-induced cytotoxicity, radioactivity was corrected for the number of viable cells, counted using trypan blue, and expressed as counts/min per 100,000 cells. Binding assays were performed in triplicate as a single assay. For modulation experiments, tumor cell lines were treated with $10 \mu \mathrm{M}$ of the mitogen-activated protein kinase kinase 1/2 inhibitor selumetinib (AZD6244; Axon Medchem) for $24 \mathrm{~h}$ before analysis. Modulation experiments were performed in 3 independent biologic replicates.

\section{Animal Studies}

Animal studies were performed according to the Dutch Regulations for Animal Welfare. The protocol was approved by the animal ethical committee of the University of Groningen. To assess tracer binding in vivo, 5- to 8-wk-old immune-deficient BALB/c nude mice (BALB/ cOlaHsd-Foxn1nu; Envigo) were subcutaneously inoculated with tumor cells in a mixture of Matrigel (high protein concentration; Corning) and PBS (H292, $5 \times 10^{6}$ in $300 \mu \mathrm{L}$ of Matrigel/PBS (1:1);
$\mathrm{H} 358,2 \times 10^{6}$ in $300 \mu \mathrm{L}$ of Matrigel/PBS (1:1); ES2, $5 \times 10^{6}$ in 300 $\mu \mathrm{L}$ of PBS). Tumor size and animal weight were measured 2 times per week. When tumors were $100-200 \mathrm{~mm}^{3}$, treatment studies and PET imaging studies were performed.

\section{Treatment Studies}

Mice xenografted with $\mathrm{H} 292$ cells were randomized between vehicle (diluent only) and IFN $\gamma$ treatment. Recombinant human IFN $\gamma$ was administered at different daily doses $\left(1 \times 10^{3}, 1 \times 10^{4}\right.$, and $1 \times 10^{5}$ IU, corresponding to $0.05,0.5$, and $5 \mu \mathrm{g} ; 5-6$ mice per group) by intraperitoneal injection once daily for $3 \mathrm{~d}$. On day 3, PET imaging studies were performed.

Mice xenografted with ES2 cells were randomized between vehicle treatment (diluent only) and selumetinib treatment groups (4-5 mice per group). Selumetinib was diluted in $0.2 \%$ polysorbate- 80 and $0.5 \%$ hydroxyl-propyl methylcellulose (Sigma-Aldrich) and administered at $10 \mathrm{mg} / \mathrm{kg}$ twice daily by oral gavage, starting $24 \mathrm{~h}$ before PET scanning. The dosage was based on earlier research indicating minimal effects on tumor growth at this concentration (18).

\section{PET Studies}

For PET imaging, ${ }^{18} \mathrm{~F}-\mathrm{BMS}-986192(8.20 \pm 4.74 \mathrm{MBq}$; average, 3 $\mathrm{MBq} / \mu \mathrm{g}$ ) was injected intravenously via the penile vein. Immediately after tracer injection, a 60-min dynamic PET scan was performed. The mice were placed in a Focus 220 rodent scanner (CTI Siemens) and kept warm on heating mats. After the emission scan, a transmission scan of $515 \mathrm{~s}$ was performed using a ${ }^{57} \mathrm{Co}$ point source to correct for scatter, random coincidences, and tissue attenuation. After the scan, the mice were killed for an ex vivo biodistribution analysis. Organs were dissected and weighed, and their radioactivity was measured in a $\gamma$-counter. Uptake in the organs was calculated as percentage injected dose per gram of tissue (\% ID/g).

\section{PET Reconstruction}

PET data were reconstructed into 10-min frames, and in vivo quantification was performed using PMOD software (version 4.0; PMOD Technologies LCC). Three-dimensional regions of interest were drawn around the tumor, based on ex vivo measured tumor volume, and a threshold equal to $40 \%$ of the maximum intensity was used to determine uptake. Necrotic parts of the tumor were excluded. For other organs, a fixed-size sphere was drawn in representative parts of the organs. Tracer uptake was calculated as the average uptake in the last frame (50-60 min after injection) and presented as \% ID/g, based on previous results (16).

\section{Ex Vivo and In Vitro Tumor Cell Analyses}

For PD-L1 measurements with flow cytometry, xenograft tumors were incubated overnight in RPMI 1640, with $10 \%$ fetal calf serum, $16.7 \mu \mathrm{g} / \mathrm{mL}$ DNase (Roche Diagnostics Nederland B.V.), and $1 \mathrm{mg} /$ $\mathrm{mL}$ collagenase type IV (Thermo Fischer Scientific) at room temperature. Single-cell suspensions were created using $70-\mu \mathrm{m}$ cell strainers. For in vitro PD-L1 measurements, cells growing in monolayer were harvested using trypsin. For both ex vivo and in vitro cells, 100,000 cells were stained with anti-PD-L1 (clone 29E.2A3; BioLegend) and secondary antibodies against mouse $\mathrm{IgG}$ (polyclonal goat antimouse PE; Southern Biotec) or directly stained with phycoerythrin-labeled anti-PD-L1 (MIH-I; Invitrogen). At least 10,000 events were measured on the Accuri C6 (BD Biosciences) or FACSverse (BD Biosciences) apparatus. Data analysis was based on mean fluorescence intensity. For Western blot analysis, xenograft tumors were homogenized using the Bel-Art Micro-Tube Homogenizer (Thermo Fisher Scientific). Lysates from homogenized xenograft tumors and cell lines growing in monolayer were made using a mammalian protein extraction reagent with phosphatase and protease inhibitors diluted 1:100 (Thermo Fisher Scientific). Proteins were separated using sodium 
dodecyl sulfate polyacrylamide gel electrophoresis. Membranes were stained with 1:1,000 rabbit anti-PD-L1 (E1L3N; Cell Signaling Technology), glyceraldehyde 3-phosphate dehydrogenase (EPR6256; Abcam), and secondary horseradish peroxidase-antimouse or horseradish peroxidaseantirabbit antibodies at 1:1,500 (Dako). Detection was performed using Lumi-Light Western blotting substrate (Roche Diagnostics Nederland B.V.) and a digital imaging system (Bio-Rad). Western blot signals were quantified by measuring the relative optical density of the target protein, compared with the relative glyceraldehyde 3-phosphate dehydrogenase optical density using imageJ after subtraction of the background signal.

For immunohistochemistry, formalin-fixed paraffin-embedded xenograft tumors were cut into $4-\mu \mathrm{m}$ slices and placed on glass slides. Antigen was retrieved using universal heat-induced epitope retrieval reagent (Abcam), followed by endogenous peroxidase block (S2003; Dako), endogenous IgG block (X0909; Dako), and incubation with antiPD-L1 antibody (clone 28-8; Abcam) for $60 \mathrm{~min}$. Next, sections were incubated with antirabbit Dako EnVision+ polymer for 30 min (K4010; Dako). The staining was visualized using 3,3'-diaminobenzidine + substrate (K3468; Dako) and counterstained using hematoxylin.

\section{Statistical Analysis}

Data are presented as mean $\pm \mathrm{SD}$. A $t$ test, Kruskal-Wallis test with Dunn multiple-comparison test, or ANOVA with Bonferroni multiplecomparison test was performed to compare groups (GraphPad; Prism 7). $P$ values of less than 0.05 were considered statistically significant.

\section{RESULTS}

\section{F-BMS-986192 Binding Increases with Higher PD-L1 \\ Expression}

To study the ability of ${ }^{18}$ F-BMS-986192 to detect a range of PD-L1 levels, we selected 4 tumor cell lines with different basal PD-L1 membrane expression levels in vitro as measured by flow cytometry (Fig. 1A). Binding assays confirmed 2-fold increased binding of ${ }^{18} \mathrm{~F}-\mathrm{BMS}-986192$ to H358 cells, compared with H292 cells, corresponding to the difference in basal PD-L1 expression (Supplemental Fig. 1A). Tracer binding could be blocked by adding nonradioactive ${ }^{19} \mathrm{~F}-\mathrm{BMS}-986192$, indicating specific binding (Supplemental Fig. 1B). In vivo PET experiments were performed with xenograft models of H292, H358, and ES2. ${ }^{18}$ F-BMS-986192 uptake in the different xenograft models when measured with PET imaging (\%ID/g $\pm \mathrm{SD}: \mathrm{H} 292,1.33 \pm 0.37$; $\mathrm{H} 358,1.62 \pm 0.86$; ES2, $2.36 \pm 1.07)$ and ex vivo biodistribution $(\% \mathrm{ID} / \mathrm{g} \pm \mathrm{SD}: \mathrm{H} 292$, $1.64 \pm 0.60 ; \mathrm{H} 358,2.81 \pm 1.84$; ES2, $4.25 \pm$ 2.3) was associated with PD-L1 expression (Figs. 1B and 1C). Western blot and immunohistochemical analysis confirmed the differential PD-L1 expression levels in vivo, with ES2 showing the highest PD-L1 levels (Figs. 1D and 1E). Tracer levels in other organs were low, except at the site of renal excretion (Supplemental Figs. 1C and 1D).

\section{${ }^{18}$ F-BMS-986192 Tumor Uptake Reflects Failure of IFNy to Induce PD-L1 Expression In Vivo}

We have previously demonstrated that the proinflammatory cytokine IFN $\gamma$ activates the IFN $\gamma$ signaling pathway and increases fluorescence intensity. both total protein and membrane levels of PD-L1 in H292 and H358 cells in vitro (19). In the present study, these cell lines showed increased tracer binding after $24 \mathrm{~h}$ of treatment with IFN $\gamma$ in vitro (Supplemental Fig. 2A). Both in vivo PET results and ex vivo analyses showed a minor trend toward higher ${ }^{18}$ F-BMS-986192 uptake in $\mathrm{H} 292$ tumors after treatment of the mice with up to $10^{5}$ IU of IFN $\gamma$ daily for $3 \mathrm{~d}$ (Fig. 2A). Treatment did not influence tumor volume. In other organs, no difference in tracer distribution was observed (Supplemental Figs. 2B and 2C). Western blot and immunohistochemical analysis confirmed that IFN $\gamma$ treatment failed to increase PD-L1 expression in tumors, with only a nonsignificant increase in IFN $\gamma$-signaling protein pSTAT1, a known activator of PD-L1 transcription (Figs. 2B and 2C).

\section{F-BMS-986192 Tumor Uptake Reflects Failure of Selumetinib to Reduce Tumor Cell PD-L1 Membrane Expression Levels In Vivo}

Next, we investigated whether ${ }^{18}$ F-BMS-986192 could detect treatment-induced downregulation of PD-L1. In vitro treatment of the strongly PD-L1-positive ES2 cells with selumetinib for $24 \mathrm{~h}$ resulted in a complete blockade of mitogen-activated protein kinase kinase $1 / 2$ signaling and approximately $50 \%$ downregulation of PD-L1 membrane expression levels and total PD-L1 protein expression (Fig. 3A). In vivo and ex vivo analyses showed that $1 \mathrm{~d}$ of treatment with $10 \mathrm{mg} / \mathrm{kg}$ selumetinib by oral gavage did not alter ${ }^{18}$ F-BMS-986192 tumor uptake or biodistribution compared with vehicle-treated mice (Figs. 3B; Supplemental Figs. 3A and 3B). Moreover, flow cytometric analysis confirmed that selumetinib treatment did not lead to a reduction of tumor cell PD-L1 membrane expression levels (Fig. 3C). Ex vivo analysis, however, showed that the treatment effectively inhibited mitogen-activated protein kinase kinase 1/2 signaling and cellular PD-L1 expression (Figs. 3D and 3E). Treatment did not influence tumor volume. These

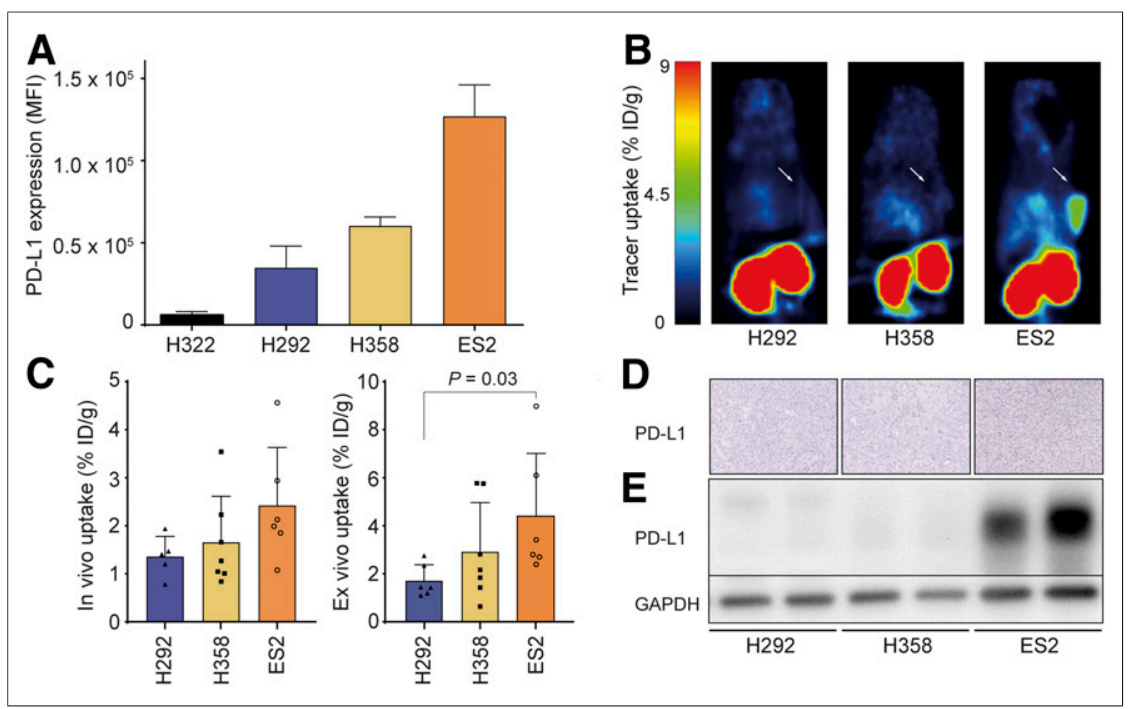

FIGURE 1. PD-L1 expression correlates with tracer uptake. (A) Basal PD-L1 membrane expression of panel of in vitro cultured cell lines as determined with flow cytometry. (B) In vivo tracer distribution in \%ID/g 60 min after injection of ${ }^{18} \mathrm{~F}-\mathrm{BMS}-986192$ in BALB/c nude mice with subcutaneous H292, H358, or ES2 tumors. White arrow indicates location of tumor. (C) In vivo and ex vivo ${ }^{18} \mathrm{~F}-\mathrm{BMS}-986192$ uptake in xenografts $60 \mathrm{~min}$ after tracer injection. (D and $\left.\mathrm{E}\right)$ Tumor PD-L1 expression levels as analyzed using immunohistochemistry (D) and Western blotting (E). Data are presented as mean + SD. GAPDH = glyceraldehyde 3-phosphate dehydrogenase; MFI = mean 


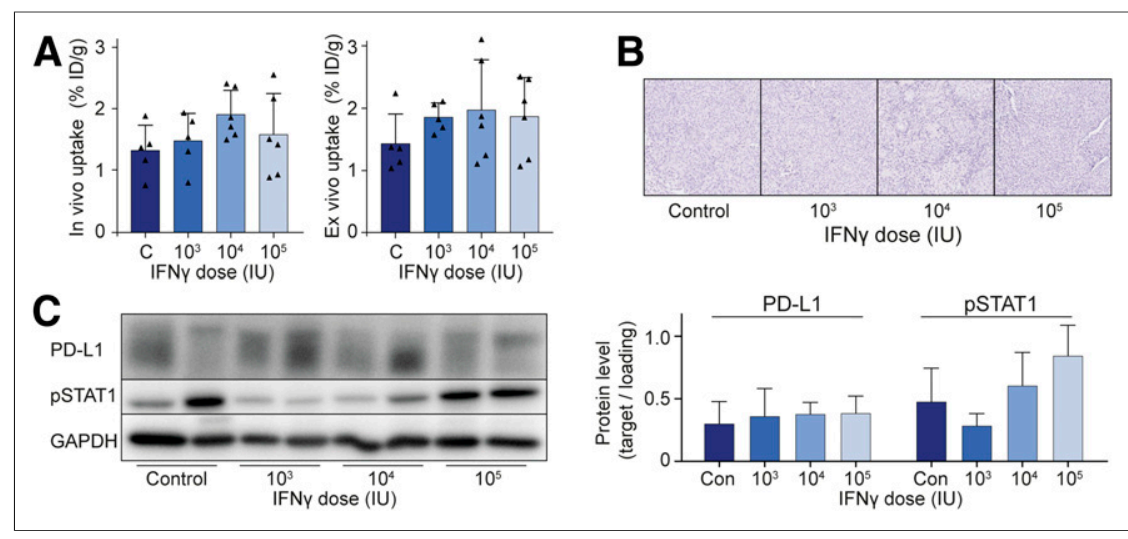

FIGURE 2. IFNy fails to induce PD-L1 expression in vivo. (A) In vivo and ex vivo tracer uptake in $\% I D / g 60$ min after injection of ${ }^{18} \mathrm{~F}-\mathrm{BMS}-986192$ in BALB/c nude mice, with subcutaneous $\mathrm{H} 292$ xenografts treated with IFNy once daily for $3 \mathrm{~d}$ by intraperitoneal injection. (B and C) H292 xenograft PD-L1 expression after treatment with different doses of IFNy as measured using immunohistochemistry (B) and Western blotting (C). PD-L1 and pSTAT1 were measured in triplicate and quantified relative to glyceraldehyde 3-phosphate dehydrogenase (GAPDH). Data are presented as mean $+\mathrm{SD}$. C and Con = control; IU = international units.

results indicate that ${ }^{18} \mathrm{~F}-\mathrm{BMS}-986192$ specifically detects PD-L1 membrane levels and that tracer uptake is not influenced by cellular PD-L1 levels.

\section{DISCUSSION}

In this study, we assessed the utility of ${ }^{18} \mathrm{~F}-\mathrm{BMS}-986192$ PET to noninvasively measure PD-L1 expression levels. ${ }^{18} \mathrm{~F}-\mathrm{BMS}-986192$ tumor uptake was related to basal PD-L1 expression in cell lines and in xenograft models, enabling noninvasive detection of differential PD-L1 membrane levels. Selumetinib treatment reduced cellular PD-L1 expression. However, PD-L1 membrane levels were not altered by treatment with IFN $\gamma$ or selumetinib. Tracer uptake was not affected by treatment, suggesting that ${ }^{18} \mathrm{~F}-\mathrm{BMS}-986192$ PET reflects membrane levels, rather than cellular expression, of PD-L1.

To our knowledge, this was the first study demonstrating that same-day PET imaging with an adnectin-based tracer discriminates low from moderate and high tumor cell PDL1 expression using preclinical in vivo models. ${ }^{18}$ F-BMS-986192 can detect 2-fold differences in PD-L1 membrane levels. Tumor uptake of ${ }^{18} \mathrm{~F}-\mathrm{BMS}-986192$, expressed as $\% \mathrm{ID} / \mathrm{g}$, is lower than uptake with monoclonal antibody-based tracers, although similar to results in other preclinical studies evaluating same-day imaging of PD-L1 (2024). ${ }^{18} \mathrm{~F}-\mathrm{BMS}-986192$ is specific for human PD-L1, with picomolar dissociation constants $(<35 \mathrm{pM})$. This prevents mouse tissues from influencing tracer uptake (16). However, in humanized immune-competent models, with human PD-L1-expressing immune cells, tracer distribution might be different. Moreover, in these models and in patients, tracer uptake in the tumor might differ because of gen-activated protein kinase; Selu = selumetinib. increased tumor cell PD-L1 expression and PD-L1-positive tumor immune cell infiltration (25).

To study imaging of treatment-induced changes in PD-L1 expression, we aimed to modulate PD-L1 expression levels in tumor xenografts. IFN $\gamma$, a well-known inducer of tumor cell PD-L1 expression in vitro (26), failed to increase tumor cell PD-L1 expression in vivo. Although intravenous administration of IFN $\gamma$ increases mouse lung PD-L1 expression and intraperitoneal administration of IFN $\gamma$ sensitizes xenograft models for pemetrexed, direct evidence of tumor cell PD-L1 upregulation by IFN $\gamma$ in vivo is sparse $(4,27,28)$. Because PD-L1 expression is regulated on many levels, it may be that factors critical for PD-L1 upregulation are missing in the tumor microenvironment of H292 xenografts (4). Additionally, we aimed to reduce PD-L1 expression of tumors by treatment with selumetinib. Despite reduced total PD-L1 protein levels, PD-L1 membrane levels and tumor ${ }^{18}$ F-BMS-986192 uptake were not affected after treatment in vivo. Multiple posttranslational mechanisms, including altered recycling of PD-L1 to the cell membrane, may give rise to the lack of correlation between total PD-L1 protein levels and PD-L1 membrane levels (2932). In our model, the reduction of total protein levels in vivo may be followed by a delayed reduction in PD-L1 membrane levels, after the 24-h time point. In addition, tracer uptake corresponded better with PD-L1 membrane expression than with total protein levels of PD-L1 (Supplemental Fig. 4). These modulation experiments show the

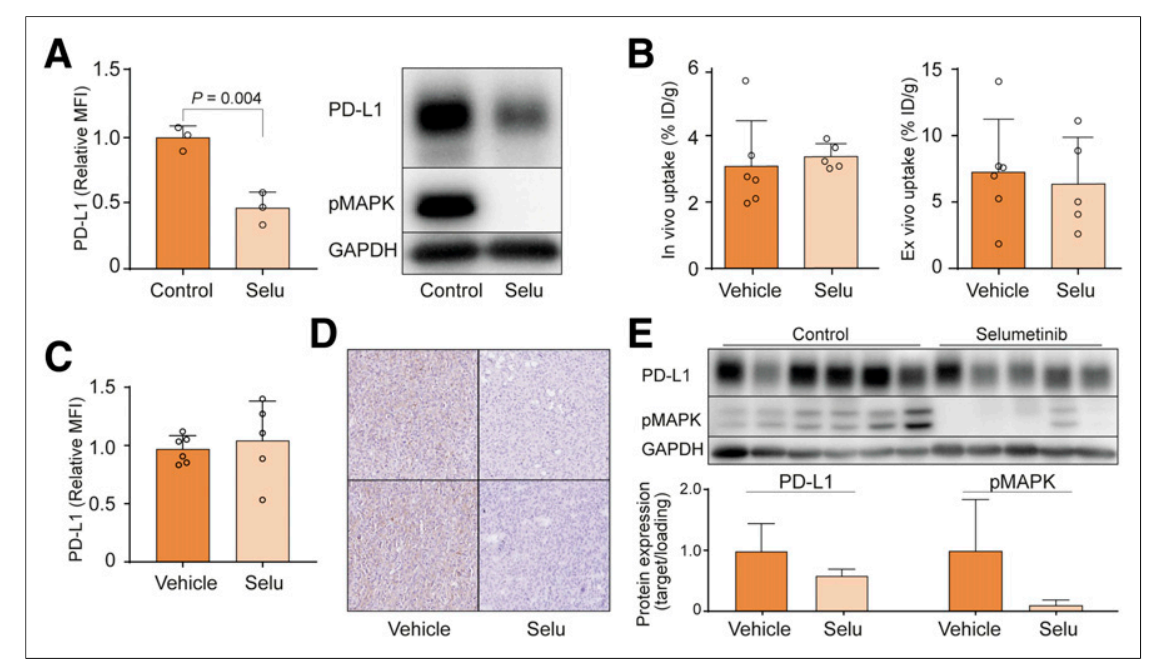

FIGURE 3. Selumetinib decreased PD-L1 expression in vitro and in vivo. (A) ES2 cells were treated in vitro with $10 \mu \mathrm{M}$ selumetinib for $24 \mathrm{~h}$, followed by flow cytometry and Western blotting of PD-L1 membrane and total protein expression and MAPK signaling. (B) In vivo and ex vivo tracer uptake in \%ID/g 60 min after injection of ${ }^{18} \mathrm{~F}-\mathrm{BMS}-986192$ in BALB/c nude mice with subcutaneous ES2 tumors and treated 2 times with $10 \mathrm{mg} / \mathrm{kg}$ selumetinib for $24 \mathrm{~h}$ by oral gavage. (C-E) Tumor cell PD-L1 levels as measured using flow cytometry (C), immunohistochemistry (D), and Western blotting (E). Additionally, PMAPK and PD-L1 were quantified by measuring their signal relative to glyceraldehyde 3-phosphate dehydrogenase (GAPDH) signal. Data are presented as mean + SD. MFI = mean fluorescence intensity; pMAPK = phosphorylated mito- 
challenges of studying immune checkpoint biology in preclinical models. The complex interaction between cell types in the tumor microenvironment is suboptimally reflected in mouse models, urging critical evaluation of clinical validity of preclinical findings. However, we were still able to detect different tumor cell PD-L1 levels, indicating that serial imaging with this tracer in patients may provide insight into treatment effects on PD-L1 membrane levels. Interestingly, most approved PD-L1 immunohistochemical assays measure the membrane-bound fraction of PD-L1, because this is hypothesized to be immunologically most active (33).

Clinically, PD-L1 imaging has the potential to predict response to immunotherapy better than PD-L1 immunohistochemistry, as shown in the first-in-human clinical study with the anti-PD-L1 monoclonal antibody ${ }^{89} \mathrm{Zr}$-atezolizumab (34). Comparing ${ }^{89} \mathrm{Zr}$ atezolizumab imaging with a clinical study using ${ }^{18} \mathrm{~F}-\mathrm{BMS}$ 986192, results suggest that this PD-L1 antibody-based PET tracer reaches higher target uptake levels than ${ }^{18} \mathrm{~F}$-BMS-986192 (34,35). However, the short serum half-life and rapid diffusion of ${ }^{18} \mathrm{~F}$ BMS-986192 enable same-day imaging with high-contrast images and reduced radiation burden $(35,36)$. This advantage might allow rapid follow-up of treatment-induced changes in PD-L1 expression with PET imaging. Also, it enables combined PET imaging of multiple targets on subsequent days, providing more information. The feasibility of imaging multiple immunotherapy-related targets in a single patient was shown in a small study using ${ }^{18} \mathrm{~F}-\mathrm{BMS}$ 986192 in combination with PD-1-targeting ${ }^{89} \mathrm{Zr}$-nivolumab in 13 patients (35). Currently, ${ }^{18} \mathrm{~F}-\mathrm{BMS}-986192$ is being further investigated in metastatic melanoma, non-small cell lung cancer, and oral cancer. ${ }^{18} \mathrm{~F}$-BMS-986192 PET imaging is performed at baseline and during nivolumab treatment (ClinicalTrials.gov identifiers NCT03520634 and NCT03843515). In both studies, PET uptake will be correlated with PD-L1 expression in biopsy samples measured by immunohistochemistry. Future larger studies will have to define the precise role of PET imaging in predicting tumor response to immune checkpoint inhibitors or to study treatment effects on PD-L1 expression by performing serial imaging.

\section{CONCLUSION}

${ }^{18}$ F-BMS-986192 PET imaging can be used to noninvasively quantify PD-L1 membrane levels. This ability makes it a potential tool to study PD-L1 expression dynamics and predict responses to immunotherapy. Further clinical evaluation will be necessary to validate these findings in humans.

\section{DISCLOSURE}

Erik de Vries reports grants from ZonMW (grants 95104008 and 95105010) and from the Dutch Cancer Foundation (grant RUG2015-7235) during the conduct of the study and has performed contract research studies with Rodin Therapeutics, Lysosomal Therapeutics Inc., Hoffmann-La Roche Ltd., and Ionis Pharmaceuticals, with funds made available to the institution outside the submitted work. Geke Hospers reports a consulting and advisory role for Amgen, Roche, MSD, BMS, Pfizer, and Novartis and has received grants from BMS and Seerave, made available to the institution outside the submitted work. Elisabeth de Vries reports grants from IMI TRISTAN (GA grant 116106) during the conduct of the study; has a consulting and advisory role for NSABP, Daiichi Sankyo, Pfizer, Sanofi, Merck, and Synthon Biopharmaceuticals; and has received grants from Amgen, Genentech, Roche, Chugai Pharma, CytomX Therapeutics, Nordic Nanovector, G1 Therapeutics, AstraZeneca, Radius Health, and Bayer, all made available to the institution outside the submitted work. Cooperators at Bristol Meyers Squibb approved the preclinical design and the final version of the manuscript. Thijs S. Stutvoet is supported by a fellowship of the Junior Scientific Master Class of the University of Groningen. No other potential conflict of interest relevant to this article was reported.

\section{ACKNOWLEDGMENTS}

We thank Ralph Adam Smith, Wendy Hayes, David Leung, David J. Donnelly, Samuel J. Bonacorsi, and Paul Morin for their efforts to support the work performed during this study.

\section{KEY POINTS}

QUESTION: Can ${ }^{18}$ F-BMS-986192 PET be used to detect different PD-L1 expression levels and therapy-induced changes in tumor cell PD-L1 expression?

PERTINENT FINDINGS: ${ }^{18}$ F-BMS-986192 PET imaging in immune-competent tumor-bearing mice allows detection of PD-L1 membrane levels as soon as $60 \mathrm{~min}$ after tracer injection. The tracer can discriminate a range of tumor cell PD-L1 membrane levels.

IMPLICATIONS FOR PATIENT CARE: $18 \mathrm{~F}-\mathrm{BMS}-986192$ PET imaging might be a potential tool to study PD-L1 expression dynamics and predict responses to immunotherapy in humans.

\section{REFERENCES}

1. Ribas A, Wolchok JD. Cancer immunotherapy using checkpoint blockade. Science. 2018;359:1350-1355.

2. Hodi FS, Chiarion-Sileni V, Gonzalez R, et al. Nivolumab plus ipilimumab or nivolumab alone versus ipilimumab alone in advanced melanoma (CheckMate 067): 4-year outcomes of a multicentre, randomised, phase 3 trial. Lancet Oncol. 2018;19:1480-1492.

3. Tang J, Shalabi A, Hubbard-Lucey VM. Comprehensive analysis of the clinical immuno-oncology landscape. Ann Oncol. 2018;29:84-91.

4. Sun C, Mezzadra R, Schumacher TN. Regulation and function of the PD-L1 checkpoint. Immunity. 2018;48:434-452.

5. Nishijima TF, Shachar SS, Nyrop KA, Muss HB. Safety and tolerability of PD-1/ PD-L1 inhibitors compared with chemotherapy in patients with advanced cancer: a meta-analysis. Oncologist. 2017;22:470-479.

6. Gong J, Chehrazi-Raffle A, Reddi S, Salgia R. Development of PD-1 and PD-L1 inhibitors as a form of cancer immunotherapy: a comprehensive review of registration trials and future considerations. J Immunother Cancer. 2018;6:8.

7. Broos K, Lecocq Q, Raes G, Devoogdt N, Keyaerts M, Breckpot K. Noninvasive imaging of the PD-1: PD-L1 immune checkpoint: embracing nuclear medicine for the benefit of personalized immunotherapy. Theranostics. 2018;8:3559-3570.

8. Carbognin L, Pilotto S, Milella M, et al. Differential activity of nivolumab, pembrolizumab and MPDL3280A according to the tumor expression of programmed death-ligand-1 (PD-L1): sensitivity analysis of trials in melanoma, lung and genitourinary cancers. PLoS One. 2015;10:e130142.

9. Madore J, Vilain RE, Menzies AM, et al. PD-L1 expression in melanoma shows marked heterogeneity within and between patients: implications for anti-PD-1/ PD-L1 clinical trials. Pigment Cell Melanoma Res. 2015;28:245-253.

10. Uruga H, Bozkurtlar E, Huynh TG, et al. Programmed cell death ligand (PD-L1) expression in stage II and III lung adenocarcinomas and nodal metastases. $J$ Thorac Oncol. 2017;12:458-466.

11. Munari E, Zamboni G, Marconi M, et al. PD-L1 expression heterogeneity in nonsmall cell lung cancer: evaluation of small biopsies reliability. Oncotarget. 2017;8: 90123-90131.

12. Sheng J, Fang W, Yu J, et al. Expression of programmed death ligand-1 on tumor cells varies pre and post chemotherapy in non-small cell lung cancer. Sci Rep. 2016;6:20090.

13. Bailly C, Cléry P-F, Faivre-Chauvet A, et al. Immuno-PET for clinical theranostic approaches. Int J Mol Sci. 2016;18:57.

14. van der Veen EL, Bensch F, Glaudemans AWJM, Lub-de Hooge MN, de Vries EGE. Molecular imaging to enlighten cancer immunotherapies and underlying involved processes. Cancer Treat Rev. 2018;70:232-244. 
15. Lipovsek D. Adnectins: engineered target-binding protein therapeutics. Protein Eng Des Sel. 2011;24:3-9.

16. Donnelly DJ, Smith RA, Morin P, et al. Synthesis and biologic evaluation of a novel ${ }^{18} \mathrm{~F}$-labeled adnectin as a PET radioligand for imaging PD-L1 expression. $J$ Nucl Med. 2018;59:529-535.

17. Lee S-J, Jang B-C, Lee S-W, et al. Interferon regulatory factor-1 is prerequisite to the constitutive expression and IFN-gamma-induced upregulation of B7-H1 (CD274). FEBS Lett. 2006;580:755-762.

18. Caumanns JJ, van Wijngaarden A, Kol A, et al. Low-dose triple drug combination targeting the PI3K/AKT/mTOR pathway and the MAPK pathway is an effective approach in ovarian clear cell carcinoma. Cancer Lett. 2019;461:102-111.

19. Stutvoet TS, Kol A, de Vries EG, et al. MAPK pathway activity plays a key role in PD-L1 expression of lung adenocarcinoma cells. J Pathol. 2019;249:52-64.

20. González Trotter DE, Meng X, McQuade P, et al. In vivo imaging of the programmed death ligand 1 by ${ }^{18}$ F PET. $J$ Nucl Med. 2017;58:1852-1857.

21. Natarajan A, Patel CB, Ramakrishnan S, Panesar PS, Long SR, Gambhir SS. A novel engineered small protein for positron emission tomography imaging of human programmed death ligand-1: validation in mouse models and human cancer tissues. Clin Cancer Res. 2019;25:1774-1785.

22. Kumar D, Lisok A, Dahmane E, et al. Peptide-based PET quantifies target engagement of PD-L1 therapeutics. J Clin Invest. 2019;129:616-630.

23. Lv G, Sun X, Qiu L, et al. PET imaging of tumor PD-L1 expression with a highly specific nonblocking single-domain antibody. J Nucl Med. 2020;61:117-122.

24. Maute RL, Gordon SR, Mayer AT, et al. Engineering high-affinity PD-1 variants for optimized immunotherapy and immuno-PET imaging. Proc Natl Acad Sci USA. 2015;112:E6506-E6514.

25. Broos K, Keyaerts M, Lecocq Q, et al. Non-invasive assessment of murine PDL1 levels in syngeneic tumor models by nuclear imaging with nanobody tracers. Oncotarget. 2017;8:41932-41946.
26. Garcia-Diaz A, Shin DS, Moreno BH, et al. Interferon receptor signaling pathways regulating PD-L1 and PD-L2 expression. Cell Rep. 2017;19:1189-1201.

27. Maleki Vareki S, Chen D, Di Cresce C, et al. IDO downregulation induces sensitivity to pemetrexed, gemcitabine, FK866, and methoxyamine in human cancer cells. PLoS One. 2015;10:e0143435.

28. Hettich M, Braun F, Bartholomä MD, Schirmbeck R, Niedermann G. Highresolution PET imaging with therapeutic antibody-based PD-1/PD-L1 checkpoint tracers. Theranostics. 2016;6:1629-1640.

29. Li C-W, Lim S-O, Xia W, et al. Glycosylation and stabilization of programmed death ligand-1 suppresses T-cell activity. Nat Commun. 2016;7:12632.

30. Horita H, Law A, Hong S, Middleton K. Identifying regulatory posttranslational modifications of PD-L1: a focus on monoubiquitination. Neoplasia. 2017;19: 346-353.

31. Mezzadra R, Sun C, Jae LT, et al. Identification of CMTM6 and CMTM4 as PDL1 protein regulators. Nature. 2017;549:106-110.

32. Varthaman A, Moreau HD, Maurin M, Benaroch P. TLR3-induced maturation of murine dendritic cells regulates CTL responses by modulating PD-L1 trafficking. PLoS One. 2016;11:e0167057.

33. Wu Y, Chen W, Xu ZP, Gu W. PD-L1 distribution and perspective for cancer immunotherapy: blockade, knockdown, or inhibition. Front Immunol. 2019;10:2022.

34. Bensch F, van der Veen EL, Lub-de Hooge MN, et al. ${ }^{89} \mathrm{Zr}$-atezolizumab imaging as a non-invasive approach to assess clinical response to PD-L1 blockade in cancer. Nat Med. 2018;24:1852-1858.

35. Niemeijer AN, Leung D, Huisman MC, et al. Whole body PD-1 and PD-L1 positron emission tomography in patients with non-small-cell lung cancer. Nat Commun. 2018;9:4664.

36. Magzoub M, Jin S, Verkman AS. Enhanced macromolecule diffusion deep in tumors after enzymatic digestion of extracellular matrix collagen and its associated proteoglycan decorin. FASEB J. 2008;22:276-284.

\section{Erratum}

In the article "Ventricular Tachycardia (VT) Substrate Characteristics: Insights from Multimodality Structural and Functional Imaging of the VT Substrate Using Cardiac MRI Scar, ${ }^{123}$ I-Metaiodobenzylguanidine SPECT Innervation, and Bipolar Voltage, by Imanli et al. (J Nucl Med. 2019;60:79-85), an affiliation for author Yousra Ghzally was inadvertently left off of the affiliation list. The affiliations for Dr. Yousra Ghzally should have included: Assiut University, Arab Republic of Egypt. The authors regret the error. 